\title{
Salvage radiation therapy for postoperative locoregionally recurrent non-small cell lung cancer: a single-center experience
}

\author{
Yoon Young Jo, Su Ssan Kim, Si Yeol Song, Eun Kyung Choi \\ Department of Radiation Oncology, Asan Medical Center, University of Ulsan College of Medicine, Seoul, Korea
}

Received: July 20, 2021

Revised: September 8, 2021

Accepted: September 9, 2021

\section{Correspondence:}

Su Ssan Kim

Department of Radiation Oncology, Asan Medical Center, University of Ulsan College of Medicine, 88 Olympic-ro 43-gil, Songpa-gu, Seoul 05505, Korea.

Tel: +82-2-3010-5680

E-mail:watermountain@hanmail.net ORCID:

https://orcid.org/0000-0002-8473-302X
Purpose: To determine the effectiveness of salvage radiation therapy (RT) in patients with locoregional recurrence (LRR) following initial curative resection of non-small cell lung cancer (NSCLC) and identify the prognostic factors affecting survival.

Materials and Methods: Between January 2009 and January 2019, 54 patients with LRR after NSCLC surgery were treated with salvage RT (83.3\%) or concurrent chemoradiation therapy (16.7\%). Twenty-three (42.6\%), 21 (38.9\%), and 10 (18.5\%) patients had local, regional, and both recurrences, respectively. The median RT dose was 66 Gy (range, 37.5 to $70 \mathrm{~Gy}$ ). The radiation target volume included recurrent lesions with or without regional lymphatics depending on the location and recurrence type. Results: The median follow-up time from the start of RT was 28.3 months (range, 2.4 to 112.4 months) and disease-free interval (DFI) from surgery to recurrence was 21.0 months (range, 0.5 to 92.3 months). Tumor response after RT was complete response, partial response, stable disease, and progressive disease in $17,29,5$, and 3 patients, respectively. The rates of freedom from local progression at 1 and 2 years were $77.2 \%$ and $66.0 \%$, respectively. The median survival duration after RT was 24.8 months, and the 2-year overall survival (OS) rate was 51.1\%. On univariate analysis, initial stage, recurrence site, $\mathrm{DFI}$, and tumor response after RT were significant prognostic factors for OS. DFI $\geq 12$ months and tumor response after RT were statistically significant factors on multivariate Cox analysis for OS.

Conclusion: Our results demonstrated the effectiveness of salvage RT for LRR of NSCLC following curative surgery.

Keywords: Radiotherapy, Salvage therapy, Non-small cell lung cancer, Recurrence

\section{Introduction}

Lung cancer remains the leading cause of cancer-related mortality in Korea. The incidence of lung cancer ranks third in cancer statistics in Korea [1]. To date, complete surgical resection is the treatment of choice for patients with stage I, stage II, and selective stage III non-small cell lung cancer (NSCLC) [2]. Even though early diagnosis has become possible because of the development of diagnostic imaging technology and the active implementation of early screening, local recurrence remains a major problem in achieving complete remission. The reported recurrence rates after complete surgical resection have ranged from 10\% to 75\% [3-5]. The median survival duration following recurrence has been reported to be 8.1-21 months in previous studies [6,7]; therefore, post-recurrence prognosis has been generally poor. The standard treatment for locoregionally recurrent (LRR) NSCLC remains controversial. Currently, the National Comprehensive Cancer Network guideline [8] recommends local treatment modalities, including reoperation or radiation therapy (RT) with or without concurrent chemotherapy (CCRT). If resection is possible, surgery is preferred; 
however, as reported in several large series of all patients with locally recurrent disease, repeated resection with curative intent was performed in only $1.1 \%-1.7 \%$ of patients $[9,10]$. Alternatively, patients could be treated with salvage RT with or without chemotherapy. There have been several studies on salvage RT for LRR that report the median duration of overall survival (OS), ranging from 14 to 54.8 months [11-19]. However, most of the studies used the three-dimensional irradiation technique, which needs to evaluate the results when using intensity-modulated irradiation techniques that can reduce toxicity profiles with good tumor control. Patients with post-resection recurrent NSCLC treated with RT achieved the same survival outcome as newly diagnosed patients with NSCLC treated with RT with or without chemotherapy [17]. Hence, we can assume that the prognosis of patients with isolated but nonresectable regional recurrence might conceivably be equivalent to that of patients presenting with de novo nonresectable stage III disease. We speculated that patients whose tumors only recur regionally might indeed have a favorable prognosis and are possibly amenable to salvage by aggressive local treatment. We report our experience with salvage RT/CCRT for patients with LRR after curative resection for NSCLC to determine the effectiveness of salvage RT and assess prognostic factor affective survivals.

\section{Materials and Methods}

\section{Patients}

We retrospectively reviewed the medical records of patients with NSCLC who had received salvage RT after initial surgery between January 2009 and January 2019. The decision to opt for radiotherapy instead of surgical resection was made through multidisciplinary discussions, and all patients were presumed to have had a high risk of reoperation, considering the patients' condition, expected difficulty of reoperation, and risk of post-surgical morbidity and mortality. Patients enrolled in our study met the following criteria: (1) initial complete resection with or without adjuvant chemotherapy or $R T$, (2) salvage RT (CCRT) with a curative aim, (3) no clinical evidence of distant metastasis (DM), (4) adequate organ function, and (5) age $>18$ years. The diagnosis of LRR in 54 patients was based on enlarging or new lesions on chest computed tomography (CT). In some cases, the diagnosis of LRR was to be made by pathological confirmation whenever possible, either by bronchoscopic or endobronchial ultrasound-guided needle biopsy and/or aspiration cytology, which was feasible in 25 patients (46.3\%).

\section{Treatment}

RT was given using 6-MV photon beams from linear accelerators.
The gross target volume (GTV) was determined by the information from the chest CT scan and positron emission tomography-computed tomography (PET-CT) scan. The clinical target volume (CTV) was to cover the recurrent gross lesion(s) with or without covering regional lymphatics. The planning target volume (PTV) was defined as a further 5- $\mathrm{mm}$ expansion to the CTV. Three-dimensional conformal RT using 3-4 beams was mainly used in 29 patients (53.7\%), and intensity-modulated RT (IMRT) was used in 25 patients (46.3\%). The most common dose scheme was $66 \mathrm{~Gy}$ in 33 fractions. Most patients (50 of 54 patients) were treated with conventional fractionation; one patient received stereotactic body radiation therapy (SBRT) and three patients received hypofractionated RT (60 Gy/10 fx; 55 Gy/10 fx; 50 Gy/10 fx), most patients (83.3\%) were treated using RT alone. Only nine patients (16.7\%) were treated with weekly platinum-based doublet chemotherapy during the RT course. The chemotherapy regimens were weekly paclitaxel and cisplatin in six patients (11.1\%), 3-weekly etoposide and cisplatin in one patient (1.85\%), and weekly paclitaxel and carboplatin in two patients (3.7\%).

\section{Evaluation of response after treatment, toxicity, and statistical analysis}

We evaluated tumor response after salvage RT using Response Evaluation Criteria in Solid Tumors version 1.1 at 3 months post-treatment using chest CT. The failure patterns following salvage RT were subdivided into in-field failure, out-of-field regional failure, and DM. Local progression failure was defined as failure within the radiation field; locoregional failure was defined as the sum of in-field failure and out-of-field regional failure; and DM was defined as a failure in distant organs, including parenchymal pulmonary metastasis. The endpoints included the rates of freedom from local progression (FFLP), locoregional failure-free survival (LRFS), freedom from distant metastasis (FFDM), disease-free survival (DFS), and OS. The durations of FFLP, LRFS, FFDM, DFS, and OS, were calculated from the date of the start of RT to the date of the recurrence, death, or the last follow-up. During treatment and follow-up, toxicity was assessed and graded according to the Common Terminology Criteria for Adverse Events (version 5.0). Acute toxicities were defined as events that occurred up to 90 days from the end of the treatment, and late toxicities were defined as events that occurred after 90 days. The chi-square test and Student t-test were used to analyze the distributions of categorical and continuous variables, respectively. The Kaplan-Meier method was used to determine the survival rate. The log-rank test and the Cox proportional hazards regression model were used for univariate and multivariate analyses to determine prognostic risk factors, respectively. Multivariate analysis was performed on variables with a $p$-value 
$<0.2$ in univariate analysis. A p-value less than 0.05 was considered statistically significant, and SPSS version 22.0 (IBM Corporation, NY, USA) was used for the statistical analysis.

\section{Ethical statement}

This study was approved by Institutional Review Board of Asan Medical Center (No. 2021-1075) and was conducted in accordance with the principles of the Declaration of Helsinki. The requirement for informed consent was waived.

\section{Results}

\section{Patient and disease characteristics}

Fifty-four patients were included, and the characteristics of the patients and disease are listed in Tables 1 and 2, respectively. The median age was 67 years (range, 43 to 88 years), and 87\% of them were male. Of the 54 patients, 37 had at least one comorbidity. Among them, 10 patients (18.5\%) had underlying lung diseases, such as tuberculosis (including old tuberculosis), chronic obstructive pulmonary disease, asthma, and interstitial lung disease. Another 27 patients (50\%) had other underlying diseases, such as diabetes, hypertension, and hepatitis. Most of the patients showed

Table 1. Baseline characteristics of the patients $(n=54)$

\begin{tabular}{lc}
\hline Characteristic & Value \\
\hline Age (yr) & $67.3(43.0-88.0)$ \\
Sex & $47(87.0)$ \\
$\quad$ Male & $7(13.0)$ \\
Female & \\
Underlying disease & $17(31.5)$ \\
$\quad$ None & $10(18.5)$ \\
TB, COPD, asthma, ILD & $27(50.0)$ \\
Other comorbidities ${ }^{\text {a) }}$ & \\
ECOG status, before RT start & $50(92.6)$ \\
$0-1$ & $4(7.4)$ \\
$2-3$ & \\
Smoking status & $41(75.9)$ \\
(Ex-)smoker & $13(24.1)$ \\
Never smoker & \\
Alcohol use status & $33(61.1)$ \\
(Ex-)user & $21(38.9)$ \\
Never use & $21.0(0.5-92.3)$ \\
DFI (mo) & $28.3(2.4-112.4)$ \\
Follow-up (mo) &
\end{tabular}

Values are presented as median (range) or number (\%).

TB, Tuberculosis; COPD, chronic obstructive pulmonary disease; ILD, interstitial lung disease; ECOG, Eastern Cooperative Oncology Group; DFI, disease-free interval.

a) Diabetes mellitus, hypertension, hepatitis, etc. good performance of the Eastern Cooperative Oncology Group performance status $0-1$. Initial tumor histology at the time of surgery was $51.9 \%$ for squamous cell carcinoma and $48.1 \%$ for adenocarcinoma. In the initial histology grade, moderately differentiated

Table 2. Baseline characteristics of the disease

\begin{tabular}{|c|c|}
\hline Characteristic & Value \\
\hline \multicolumn{2}{|l|}{ Initial tumor histology } \\
\hline $\mathrm{SqCC}$ & $28(51.9)$ \\
\hline Adenocarcinoma & $26(48.1)$ \\
\hline \multicolumn{2}{|l|}{ Initial histology grade } \\
\hline W/D & $3(5.6)$ \\
\hline$M / D$ & $35(64.8)$ \\
\hline$P / D$ & $15(27.8)$ \\
\hline Not checkable & $1(1.9)$ \\
\hline \multicolumn{2}{|l|}{ Initial tumor stage (AJCC 8th) } \\
\hline Stage I & $25(46.3)$ \\
\hline Stage II & $11(20.4)$ \\
\hline Stage III & $18(33.3)$ \\
\hline \multicolumn{2}{|l|}{ Initial tumor location } \\
\hline Right upper lobe & $13(24.1)$ \\
\hline Right middle lobe & $1(1.8)$ \\
\hline Right lower lobe & $12(22.2)$ \\
\hline Left upper lobe & $13(24.1)$ \\
\hline Left lower lobe & $15(27.8)$ \\
\hline \multicolumn{2}{|l|}{ Initial Lung operation method } \\
\hline Lobectomy & $42(77.8)$ \\
\hline Pneumonectomy & $5(9.3)$ \\
\hline Wedge resection/segmentectomy & $7(13.0)$ \\
\hline \multicolumn{2}{|l|}{ PORT } \\
\hline History of PORT & $8(14.8)$ \\
\hline None & $46(85.2)$ \\
\hline \multicolumn{2}{|l|}{ Recurrence stage (AJCC 8th) } \\
\hline Stage I & $21(38.9)$ \\
\hline Stage II & $5(9.2)$ \\
\hline Stage III & $28(51.9)$ \\
\hline \multicolumn{2}{|l|}{ CCRT } \\
\hline CCRT with TP & $6(11.1)$ \\
\hline CCRT with EP or TC & $3(5.6)$ \\
\hline $\mathrm{RT}$ alone & 45 (83.3) \\
\hline RT dose (Gy) & $66.0(37.5-70.0)$ \\
\hline RT fraction size (Gy) & $2.2(1.8-15.0)$ \\
\hline BED $\left(\mathrm{Gy}_{10}\right)$ & $79.2(46.9-150.0)$ \\
\hline PTV volume $\left(\mathrm{cm}^{3}\right)$ & $246.3(23.9-780.0)$ \\
\hline
\end{tabular}

Values are presented as number (\%) or median (range).

SqCC, squamous cell carcinoma; W/D, well-differentiated; M/D, moderate differentiated; $P / D$, poorly differentiated; $A J C C$, American Joint Committee of Cancer; PORT, postoperative adjuvant radiation therapy; CCRT, concurrent chemoradiation therapy; TP, paclitaxel + cisplatin; EP, etoposide + cisplatin; TC, paclitaxel + carboplatin; RT, radiation therapy; $\mathrm{BED}$, biologically effective dose with $\alpha / \beta$ of $10 \mathrm{~Gy}$; PTV, planning target volume. 
cases were the most common grade (64.8\%). The initial tumor stage of the patients included in the study was stage I in 25 patients (46.3\%), stage II in 11 patients (20.4\%), and stage III in 18 patients (33.3\%). Regarding the Initial lung operation method, 42 patients (77.8\%) underwent lobectomy, 9.3\% underwent pneumonectomy, and seven patients (13\%) underwent wedge resection or segmentectomy due to poor pulmonary function test or high surgical risk. Eight patients (14.8\%) underwent postoperative adjuvant RT after surgery. The stage at recurrence varied, with recurrent stage III being the most common at 51.9\%. Most of the patients underwent salvage RT alone (45 patients, 83.3\%), and nine patients (16.7\%) underwent salvage CCRT. The median RT dose was $66 \mathrm{~Gy}$ (range, 37.5 to $70.0 \mathrm{~Gy}$ ), and the median dose converted into biologically equivalent dose with $\alpha / \beta$ of $10 \mathrm{~Gy}\left(\mathrm{BED}_{10}\right)$ was $79.2 \mathrm{~Gy}_{10}$ (range, 46.88 to $150.0 \mathrm{~Gy}_{10}$ ). The median volume of PTV was 246.3 $\mathrm{cm}^{3}$ (range, 23.9 to $780.0 \mathrm{~cm}^{3}$ ).

\section{Treatment response and survival}

The median disease-free interval (DFI) from surgery to recurrence, was 21.0 months, and it ranged from 0.5 to 92.3 months. The median follow-up period was 28.3 months (range, 2.4 to 112.4 months). Forty-six of 54 patients achieved complete response or partial response (CR in 17 patients, PR in 29 patients, stable disease in five patients, and progressive disease in three patients). OS rates at 2 years and 5 years were 51.1\% and 26.6\% (Fig. 1), and DFS rates at 1 year and 2 years were 49.9\% and 28.6\%, respectively (Fig. 2A). The median FFDM time was 63.7 months (Fig. 2B). In the case of local progression with in-field recurrence after salvage RT,

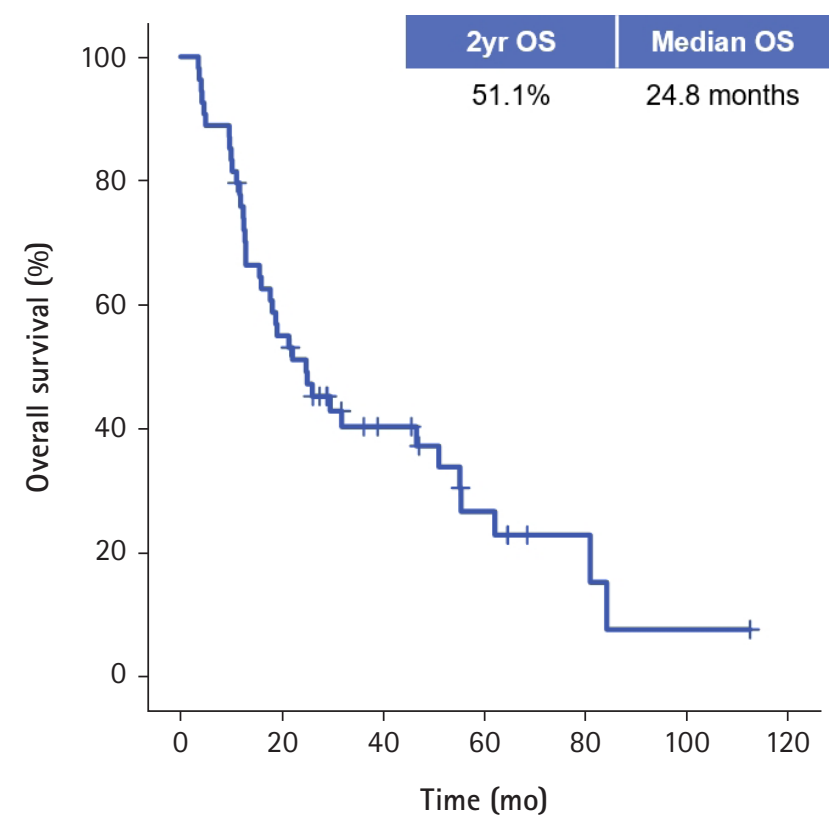

Fig. 1. Overall survival (OS) of salvage radiation therapy.
FFLP at 1 year and 2 years were 77.2\% and 66.0\%, respectively (Fig. 2C). Conversely, the median LRFS time, including both in-field and out-of-field local recurrence was 25.5 months (Fig. 2D).

The results of univariate and multivariate analyses for 2-year FFLP are presented in Table 3. RT (CCRT) response was the only significant prognostic factor for 2-year FFLP ( $p=0.014)$. In addition, as shown in Table 4, the initial stage, recurrence site, DFI, and RT (CCRT) response were significant prognostic factors for 2-year OS. In multivariate analysis, DFI longer than 12 months (hazard ratio $[\mathrm{HR}]=0.343 ; 95 \%$ confidence interval $[\mathrm{Cl}], 0.173-0.679 ; \mathrm{p}=$ $0.002)$ and $\mathrm{RT}$ (CCRT) response $(\mathrm{HR}=2.879 ; 95 \% \mathrm{Cl}, 1.235-6.709$; $\mathrm{p}=0.014$ ) were statistically significant factor for 2-year OS.

\section{Failure patterns after salvage RT}

Two-thirds of the patients $(36 / 54,66.7 \%)$ experienced recurrence after salvage RT: 16 (29.6\%) had in-field recurrence, 11 (20.4\%) had out-of-field recurrence, while 20 (37.0\%) had DM (Fig. 3). Among them, 16 patients experienced locoregional recurrence or progressive disease as their first site of recurrence, nine experienced DM as their first site of recurrence, and 11 experienced both LRR and DM as their first site of recurrence. The rates of FFLP, LRFS, and FFDM at 2 years were $66.0 \%, 50.5 \%$, and $60.6 \%$, respectively.

\section{Treatment-related RT toxicity}

Salvage RT was well tolerated with a compliance rate of $94.4 \%$; however, three patients could not finish their treatment as initially planned due to their worsening general condition. Fourteen patients (25.9\%) experienced acute radiation-induced esophagitis with grade 1 or 2 . However, there was no case of grade 3 or higher acute esophagitis. Three patients (5.56\%) had grade 3 radiation pneumonitis and were given corticosteroids, and one patient $(1.85 \%)$ was hospitalized for grade 4 radiation pneumonitis. There was no incidence of severe chronic toxicity or treatment-related mortality.

\section{Discussion and Conclusion}

The rationale for treating patients with NSCLC with locoregional relapse lies in the oligometastatic concepts. Patients with limited metastatic disease may benefit from aggressive local treatment for the macroscopic disease. Recent studies suggest that the survival of locoregional recurrent NSCLC is similar to that of stage III NSCLC than that of stage IV NSCLC $[17,18]$. Moreover, the addition of systemic therapy, including immunotherapy might improve local control.

In this retrospective study, we evaluated the effectiveness of salvage $R T$ in patients with LRR following the initial curative resection 

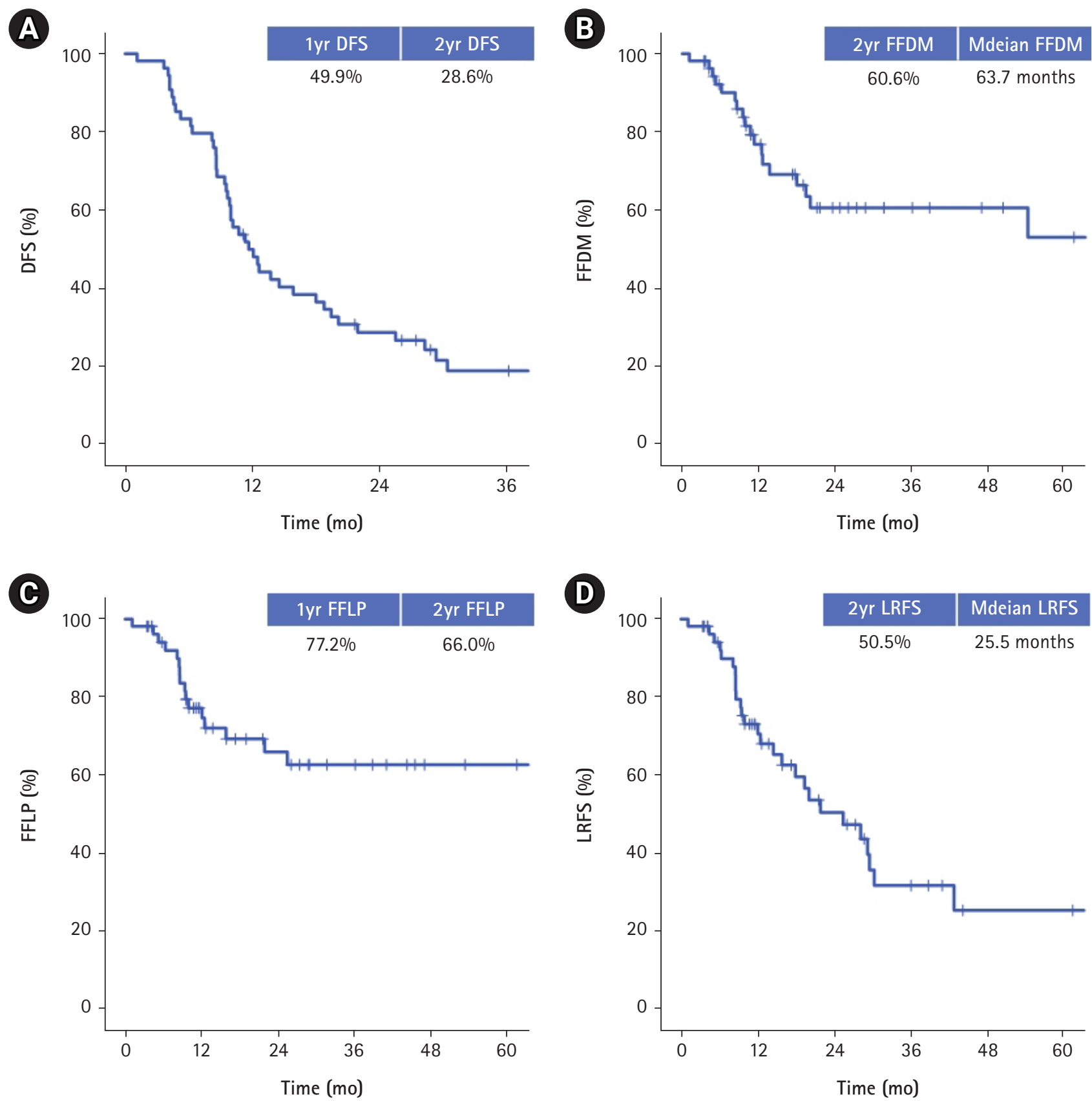

Fig. 2. Survival outcome of salvage radiation therapy. (A) Disease-free survival (DFS). (B) Freedom from distant metastasis (FFDM). (C) Freedom from local progression (FFLP). (D) Local recurrence-free survival (LRFS).

of NSCLC. The distinguishing feature of our study is that compared with previous studies (Table 5), the proportion of using IMRT was high (46.3\%). Though the benefit of IMRT has not been conclusively established in NSCLC, lower toxicity can be achieved with this technique [20]. We also included eight patients with a history of postoperative adjuvant radiation therapy (PORT) after surgery. The reason for including those patients was that we often see patients who relapse after receiving PORT in the clinic. For out-of-field recurrence, full-dose RT could be performed; thus, these patients were included.

In the case of patients with locally advanced NSCLC, several randomized trials confirmed that CCRT could significantly improve OS and progression-free survival (PFS) when compared to RT alone [21]. However, it has not been determined whether combining chemotherapy with RT can improve survival in this salvage therapy setting. Most recent and large series $(n=127)$ studies that entailed treatment with definitive CCRT suggested that concurrent chemoradiation led to significantly better outcomes than radio- 
Table 3. Univariate analysis and multivariate analysis for 2-year FFLP

\begin{tabular}{|c|c|c|c|c|c|}
\hline \multirow[b]{2}{*}{ Variable } & \multirow[b]{2}{*}{$n$} & \multicolumn{2}{|c|}{ Univariate } & \multicolumn{2}{|c|}{ Multivariate } \\
\hline & & $\begin{array}{c}2-y r \text { OS } \\
(\%)\end{array}$ & $p$-value & $\begin{array}{c}\mathrm{HR} \\
(95 \% \mathrm{Cl})\end{array}$ & $p$-value \\
\hline \multicolumn{6}{|l|}{ Age (yr) } \\
\hline$<65$ & 23 & 62 & 0.873 & - & \\
\hline$\geq 65$ & 31 & 69.6 & & & \\
\hline \multicolumn{6}{|l|}{ Histologic type } \\
\hline $\mathrm{SqCC}$ & 28 & 55.5 & 0.214 & - & \\
\hline Adenocarcinoma & 26 & 76.2 & & & \\
\hline \multicolumn{6}{|l|}{ History of PORT } \\
\hline Yes & 8 & 85.7 & 0.295 & - & \\
\hline No & 46 & 62.7 & & & \\
\hline \multicolumn{6}{|l|}{ Initial stage } \\
\hline I-II & 36 & 70.9 & 0.209 & - & \\
\hline III & 18 & 53.6 & & & \\
\hline \multicolumn{6}{|l|}{ Recurrence site } \\
\hline Stump/lung & 23 & 73.8 & 0.355 & - & \\
\hline LN/both & 31 & 58.9 & & & \\
\hline \multicolumn{6}{|l|}{ BED (Gy10) } \\
\hline$<79.2$ & 22 & 70.9 & 0.997 & - & \\
\hline$\geq 79.2$ & 32 & 62 & & & \\
\hline \multicolumn{6}{|l|}{$\mathrm{DFI}(\mathrm{mo})$} \\
\hline$<12$ & 21 & 61 & 0.227 & - & \\
\hline$\geq 12$ & 33 & 68.4 & & & \\
\hline \multicolumn{6}{|l|}{ CCRT } \\
\hline CCRT & 9 & 66.7 & 0.936 & - & \\
\hline RT alone & 45 & 64.6 & & & \\
\hline \multicolumn{6}{|l|}{ CCRT response } \\
\hline$C R, P R$ & 45 & 70.3 & 0.007 & $\begin{array}{c}4.278 \\
(1.347-13.588)\end{array}$ & 0.014 \\
\hline $\mathrm{SD}, \mathrm{PD}$ & 9 & 26.3 & & & \\
\hline \multicolumn{6}{|l|}{ Underlying disease } \\
\hline None & 17 & 67.5 & 0.99 & - & \\
\hline Yes & 37 & 64.1 & & & \\
\hline \multicolumn{6}{|l|}{ Alcohol } \\
\hline None & 21 & 80.9 & 0.127 & - & 0.227 \\
\hline (Ex-)user & 33 & 58.5 & & & \\
\hline \multicolumn{6}{|l|}{ Smoking } \\
\hline None & 13 & 71.4 & 0.715 & - & \\
\hline (Ex-)user & 41 & 64.6 & & & \\
\hline
\end{tabular}

FFLP, freedom from local progression; SqCC, squamous cell carcinoma; PORT, postoperative adjuvant radiation therapy; LN, lymph node; BED, biologically effective dose with $\alpha / \beta$ of $10 \mathrm{~Gy}$; DFl, disease-free interval; CCRT, concurrent chemoradiation therapy; $R T$, radiation therapy; $C R$, complete response; $\mathrm{PR}$, partial response; $\mathrm{SD}$, stable disease; $\mathrm{PD}$, progressive disease; $\mathrm{HR}$, hazard ratio; $\mathrm{Cl}$, confidence interval.

therapy alone [12]. However, other recent larger cohort series ( $n=$ 152) treated with definitive RT [22] did not see any clear benefit of chemotherapy on outcomes, which might be related to the fact
Table 4. Univariate analysis and multivariate analysis for 2-year OS

\begin{tabular}{|c|c|c|c|c|c|}
\hline \multirow{2}{*}{ Variable } & \multirow[b]{2}{*}{$n$} & \multicolumn{2}{|c|}{ Univariate } & \multicolumn{2}{|c|}{ Multivariate } \\
\hline & & $\begin{array}{c}2-y r \text { OS } \\
(\%)\end{array}$ & p-value & $\begin{array}{c}\mathrm{HR} \\
(95 \% \mathrm{Cl})\end{array}$ & $p$-value \\
\hline \multicolumn{6}{|l|}{ Age (yr) } \\
\hline$<65$ & 23 & 47.8 & 0.619 & - & \\
\hline$\geq 65$ & 31 & 54.1 & & & \\
\hline \multicolumn{6}{|l|}{ Histologic type } \\
\hline $\mathrm{SqCC}$ & 28 & 42.6 & 0.368 & - & \\
\hline Adenocarcinoma & 26 & 60.6 & & & \\
\hline \multicolumn{6}{|l|}{ History of PORT } \\
\hline Yes & 8 & 50 & 0.946 & - & \\
\hline No & 46 & 51.3 & & & \\
\hline \multicolumn{6}{|l|}{ Initial stage } \\
\hline$|-| \mid$ & 36 & 63.1 & 0.002 & - & 0.122 \\
\hline III & 18 & 27.8 & & & \\
\hline \multicolumn{6}{|l|}{ Recurrence site } \\
\hline Stump/lung & 23 & 64.1 & 0.042 & - & 0.749 \\
\hline LN/both & 31 & 41.9 & & & \\
\hline \multicolumn{6}{|l|}{ BED (Gy10) } \\
\hline$<79.2$ & 22 & 54.2 & 0.952 & - & \\
\hline$\geq 79.2$ & 32 & 44.8 & & & \\
\hline \multicolumn{6}{|l|}{ DFI (mo) } \\
\hline$<12$ & 21 & 28.6 & 0.003 & $\begin{array}{c}0.343 \\
(0.173-0.679)\end{array}$ & 0.002 \\
\hline$\geq 12$ & 33 & 65.7 & & & \\
\hline \multicolumn{6}{|l|}{ CCRT } \\
\hline CCRT & 9 & 49.2 & 0.978 & - & \\
\hline RT alone & 45 & 55.6 & & & \\
\hline \multicolumn{6}{|l|}{ CCRT response } \\
\hline$C R, P R$ & 45 & 56.9 & 0.007 & $\begin{array}{c}2.879 \\
(1.235-6.709)\end{array}$ & 0.014 \\
\hline$S D, P D$ & 9 & 12.5 & & & \\
\hline \multicolumn{6}{|l|}{ Underlying disease } \\
\hline None & 17 & 54.2 & 0.492 & - & \\
\hline Yes & 37 & 50 & & & \\
\hline \multicolumn{6}{|l|}{ Alcohol } \\
\hline None & 21 & 51.3 & 0.358 & - & \\
\hline (Ex-)user & 33 & 51.3 & & & \\
\hline \multicolumn{6}{|l|}{ Smoking } \\
\hline None & 13 & 51.9 & 0.942 & - & \\
\hline (Ex-)user & 41 & 51.1 & & & \\
\hline
\end{tabular}

OS, overall survival; SqCC, squamous cell carcinoma; PORT, postoperative adjuvant radiation therapy; LN, lymph node; BED, biologically effective dose with $\alpha / \beta$ of $10 \mathrm{~Gy}$; DFI, disease-free interval; CCRT, concurrent chemoradiation therapy; $\mathrm{RT}$, radiation therapy; $\mathrm{CR}$, complete response; $P R$, partial response; $S D$, stable disease; $P D$, progressive disease; $H R$, hazard ratio; $\mathrm{Cl}$, confidence interval.

that locoregional failure was a higher risk for these patients than distant metastasis. Our results are similar to those of Wu et al. [22], which reported that two-thirds of the patients experienced recur- 
Table 5. Results of salvage radiation therapy for locoregional recurrent NSCLC

\begin{tabular}{|c|c|c|c|c|c|c|}
\hline Study & Period & Number of patients & RT dose (Gy) & RT technique & CCRT (\%) & $2-y r$ OS $(\%)$ \\
\hline Kelsey et al. [11] & 1991-2003 & 29 & $66(46-74)$ & Not described & 51.7 & 38 \\
\hline \multirow[t]{2}{*}{ Lee et al. [12] } & 2004-2013 & 127 & $66(37-70)$ & 3DCRT (92.9\%) & 100 & 72.9 \\
\hline & & & & IMRT (7.1\%) & & \\
\hline Tada et al. [13] & 1992-2002 & 31 & 60 & Not described & 16.1 & 30 \\
\hline Bae et al. [14] & 1994-2007 & 64 & $54(44-66)$ & 3DCRT & 21.9 & 47.9 \\
\hline Lee et al. [15] & 2001-2009 & 38 & $60(45-75)$ & 3DCRT & 31.6 & 56 \\
\hline \multirow[t]{2}{*}{ Bar et al. [16] } & 1999-2009 & 30 & $63.5(26-66)$ & 2DCRT (57\%) & 100 & 50.8 \\
\hline & & & & 3DCRT (43\%) & & \\
\hline Cai et al. [17] & $1992-2004$ & 54 & $>59.4$ & 3DCRT & 47.8 & $14.8(5-y r)$ \\
\hline Seol et al. [18] & 2008-2013 & 31 & $66(51-66)$ & 3DCRT & 51.6 & 58.4 \\
\hline Kim et al. [19] & 2004-2014 & 57 & $66(45-70)$ & 3DCRT & 73.7 & 62.4 \\
\hline \multirow[t]{2}{*}{ Current study } & 2009-2019 & 54 & 66 (37.5-70) & 3DCRT (53.7\%) & 16.7 & 51.1 \\
\hline & & & & IMRT (46.3\%) & & \\
\hline
\end{tabular}

NSCLC, non-small cell lung cancer; RT, radiation therapy; CCRT, concurrent chemoradiation therapy; OS, overall survival; 2DCRT, two-dimensional conformal radiation therapy; 3DCRT, three-dimensional conformal radiation therapy; IMRT, Intensity-modulated radiation therapy.

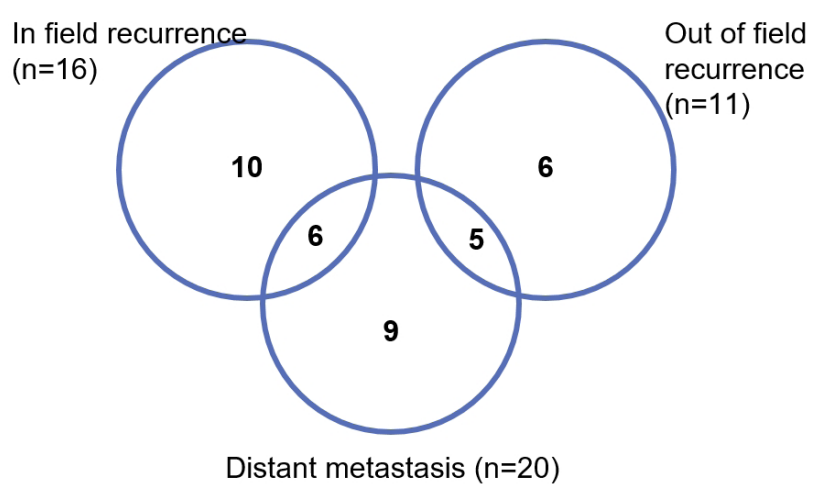

Fig. 3. Failure patterns of the first recurrence.

rence after salvage RT. Among them, 27 patients experienced locoregional failure, which was a more likely event than DM.

In terms of the radiation field, we used elective nodal irradiation for some patients with multiple lymph node (LN) station recurrences. Except for such cases, PTV covered only recurrent lesions with additional margin. The role of elective regional $L N$ irradiation in salvage RT is still unclear. For many years, the standard target volume of radical RT for primary NSCLC usually included regional nodes electively based on the high incidence of hilar and mediastinal LN metastasis. In a previous study, Kelsey et al. [11] and Tada et al. [13] used elective regional nodal irradiation for salvage RT for NSCLC. Moreover, a study [12] used CTV to cover recurrent lesions with margins without elective inclusion of regional lymphatics. However, since the CTV was set with a 5-10 mm margin on the GTV, if the recurrent lesion was along the multiple LN stations, the surrounding regional lymphatics were included, as in our case. In- dividualized radiation target volume from only recurrent lesions to elective hilar LN and mediastinal LN should be considered on a case to case basis.

Regarding the radiation dose, Jeremic et al. [23] reported there was a significant difference in the 5 -year survival rate between high-dose (55-60 Gy in 26-30 fractions) and low-dose (30 Gy in 10 fractions) RT groups. Table 5 shows the results of several studies with respect to salvage RT for LRR of NSCLC. In most studies, the median RT dose was greater than $60 \mathrm{~Gy}$. Among these studies, Bae et al. [14] reported that the low-dose group with BED $\leq 70.2 \mathrm{~Gy}_{10}$ had significantly low 2-year survival rates than the high-dose group with BED > $70.2 \mathrm{~Gy}_{10}$. And Lee et al. [12] also reported $\mathrm{BED}_{10}$ higher than $79.2 \mathrm{~Gy}_{10}$ was a significant factor for PFS. Considering that the currently recommended dose for primary NSCLC is $60-70$ Gy with conventional fractionation, it would be logical that higher doses than BED > $72 \mathrm{~Gy}_{10}$ for salvage treatment will be adequate.

Previous studies indicated that the median survival of locoregional recurrence NSCLC is comparable to that of primary NSCLC who received RT. Curran et al. [24] compared 37 patients with recurrence and 759 patients with primary NSCLC and found no significant difference in survival. Cai et al. [17] compared 54 patients with recurrence and 607 patients with primary NSCLC and reported a median survival of 20 months in the patients with recurrence, which was significantly better than that of the patients with primary NSCLC. Considering the results of the previous study, the prognosis of patients with isolated but nonresectable regional recurrence might conceivably be equivalent to that of patients presenting with de novo nonresectable stage III disease. Furthermore, the recent updated PACIFIC Trial [25] demonstrates durable PFS 
and sustained OS benefit with durvalumab after chemoradiotherapy. Consequently, a better survival outcome might be obtained if the treatment method using immunotherapy as maintenance treatment in NSCLC stage III is also implemented in patients with LRR NSCLC stage III who underwent surgery.

Our study had some limitations. First, it was a retrospective analysis. It had heterogeneous patient groups, radiation fields, and radiation dose. The results might have been affected by selection biases. Second, confirmation by biopsy of the recurrent lesion was possible in 25 patients (46.3\%). Given that most patients were diagnosed using an imaging method, there is a risk of overestimation of recurrence affecting the result of salvage treatment. Third, we evaluated radiographic tumor response by follow-up chest CT performed 3 months after the completion of salvage RT. This might be too early since response could sometimes be further prolonged, thus leading to an under-estimation of the response to salvage RT. To confirm the effectiveness of salvage RT for LRR after complete resection of NSCLC, prospective trials including a large number of patients might be needed.

In conclusion, the current study showed favorable survival outcomes after salvage RT. In the modern RT era, three-dimensional RT and IMRT appear to be effective and safe as salvage treatments for LRR after complete resection of NSCLC. Aggressive local treatment is strongly recommended for patients with LRR.

\section{Conflict of Interest}

No potential conflict of interest relevant to this article was reported.

\section{References}

1. Hong S, Won YJ, Lee JJ, et al. Cancer statistics in Korea: incidence, mortality, survival, and Prevalence in 2018. Cancer Res Treat 2021;53:301-15.

2. Zarogoulidis $K_{1}$ Zarogoulidis $P$, Darwiche $K_{1}$ et al. Treatment of non-small cell lung cancer (NSCLC). J Thorac Dis 2013;5(Suppl 4):S389-96.

3. Gilbert S, Reid KR, Lam MY, Petsikas D. Who should follow up lung cancer patients after operation? Ann Thorac Surg 2000;69:1696-700.

4. Martini N, Bains MS, Burt ME, et al. Incidence of local recurrence and second primary tumors in resected stage I lung cancer. J Thorac Cardiovasc Surg 1995;109:120-9.

5. Battafarano RJ, Piccirillo JF, Meyers BF, et al. Impact of comorbidity on survival after surgical resection in patients with stage I non-small cell lung cancer. J Thorac Cardiovasc Surg 2002;
123:280-7.

6. Sonobe $M$, Yamada $T$, Sato $M$, et al. Identification of subsets of patients with favorable prognosis after recurrence in completely resected non-small cell lung cancer. Ann Surg Oncol 2014;21: 2546-54.

7. Saisho S, Yasuda K, Maeda A, et al. Post-recurrence survival of patients with non-small-cell lung cancer after curative resection with or without induction/adjuvant chemotherapy. Interact Cardiovasc Thorac Surg 2013;16:166-72.

8. National Comprehensive Cancer Network. NCCN Guidelines for Patients: non-small cell lung cancer version 5, 2021 [Internet]. Plymouth Meeting, PA: National Comprehensive Cancer Network; c2021 [cited 2021 Sep 9]. Available from: https://www. nccn.org/guidelines/guidelines-detail?category $=1$ \&id $=1450$.

9. Gabler A, Liebig S. Reoperation for bronchial carcinoma. Thorax 1980;35:668-70.

10. Watanabe Y, Shimizu J, Oda M, Tatsuzawa Y, Hayashi Y, Iwa T. Second surgical intervention for recurrent and second primary bronchogenic carcinomas. Scand J Thorac Cardiovasc Surg 1992;26:73-8.

11. Kelsey CR, Clough RW, Marks LB. Local recurrence following initial resection of NSCLC: salvage is possible with radiation therapy. Cancer J 2006;12:283-8.

12. Lee $K H_{1}$ Ahn YC, Pyo H, et al. Salvage concurrent chemo-radiation therapy for loco-regional recurrence following curative surgery of non-small cell lung cancer. Cancer Res Treat 2019;51:769-76.

13. Tada T, Fukuda $H$, Nakagawa $K$, et al. Non-small cell lung cancer: radiation therapy for locoregional recurrence after complete resection. Int J Clin Oncol 2005;10:425-8.

14. Bae SH, Ahn YC, Nam H, et al. High dose involved field radiation therapy as salvage for loco-regional recurrence of non-small cell lung cancer. Yonsei Med J 2012;53:1120-7.

15. Lee NK, Moon SH, Kim TH, et al. Prognostic value of gross tumor volume for definitive radiation therapy in patients with locoregionally recurrent non-small-cell lung cancer after surgical resection. Clin Lung Cancer 2013;14:399-406.

16. Bar J, Ng D, Moretto P, et al. Chemoradiotherapy for locoregional recurrence of non-small-cell lung cancer after surgical resection: a retrospective analysis. Clin Lung Cancer 2013;14:200-4.

17. Cai $X W, X u L Y$, Wang $L$, et al. Comparative survival in patients with postresection recurrent versus newly diagnosed non-smallcell lung cancer treated with radiotherapy. Int J Radiat Oncol Biol Phys 2010;76:1100-5.

18. Seol KH, Lee JE, Cho JY, Lee DH, Seok Y, Kang MK. Salvage radiotherapy for regional lymph node oligo-recurrence after radical surgery of non-small cell lung cancer. Thorac Cancer 2017;8: 620-9. 
19. Kim E, Song C, Kim MY, Kim JS. Long-term outcomes after salvage radiotherapy for postoperative locoregionally recurrent non-small-cell lung cancer. Radiat Oncol J 2017;35:55-64.

20. Liao ZX, Komaki RR, Thames HD Jr, et al. Influence of technologic advances on outcomes in patients with unresectable, locally advanced non-small-cell lung cancer receiving concomitant chemoradiotherapy. Int J Radiat Oncol Biol Phys 2010;76:77581.

21. O'Rourke N, Roque I, Figuls M, Farre Bernado N, Macbeth F. Concurrent chemoradiotherapy in non-small cell lung cancer. Cochrane Database Syst Rev 2010;(6):CD002140.

22. Wu AJ, Garay E, Foster A, et al. Definitive radiotherapy for local recurrence of NSCLC after surgery. Clin Lung Cancer 2017;18: e161-e168.

23. Jeremic B, Shibamoto $Y$, Milicic $B$, et al. External beam radiation therapy alone for loco-regional recurrence of non-small-cell lung cancer after complete resection. Lung Cancer 1999;23:135-42.

24. Curran WJ Jr, Herbert SH, Stafford PM, et al. Should patients with post-resection locoregional recurrence of lung cancer receive aggressive therapy? Int J Radiat Oncol Biol Phys 1992;24:25-30.

25. Faivre-Finn C, Vicente D, Kurata T, et al. Four-year survival with durvalumab after chemoradiotherapy in stage III NSCLC: an update from the PACIFIC trial. J Thorac Oncol 2021;16:860-7. 